\title{
Complex Investigation of SBS Galaxies in Seven Selected Fields
}

\author{
Susanna Hakopian \\ Byurakan Astrophysical Observatory (BAO), Armenia \\ E-mail: sanna@web.am
}

\begin{abstract}
It is known that the main criterion for the selection of active objects in the First Byurakan, otherwise Markarian survey was the presence of signs of UV-excess in their lowdispersion spectra. Using the presence of emission lines as the second criteria became real during the Second Byurakan survey because of its improved technique. Extended (not stellated) objects, selected with the use of this criterion, made the main part of the separate sample of SBS galaxies. Originally, this sample included 1286 objects, selected in 65 fields of the survey (16 square degree each), to which, with the help of other sources than the survey, there were later added some objects. We studied a subsample of SBS galaxies in seven selected fields (the deepest according to the $\mathrm{V} / \mathrm{Vmax}$ criterion), including about the third of the whole sample. The first, already completed phase of this program was started with carrying out a follow-up slit spectroscopy of all, about 500 objects, based on observations with long-slit spectrographs with $6 \mathrm{~m}$ telescope of SAO Russia and 2.6.m telescope of Byurakan. As a result redshifts were determined, as well as spectral classification was made for all of objects, using the scheme adapted to the spectral material. Besides other, obtained data allowed us to estimate the efficiency of used criteria for the selection of galaxies of different classes of starformation and nuclear activity along the full scale of the apparent magnitudes, including close to the limit values $(18.5<$ $\left.\mathrm{m}_{p g}<19.5\right)$, etc. The fact that the total area of seven fields as the total number of objects in them comparable with these values for the survey as a whole, allows us to extrapolate the results to the whole sample of galaxies as an upper estimate. The second stage is to conduct detailed studies of individual galaxies in the first place, the most interesting in terms of morphology. They are based on panoramic spectroscopy obtained from observations at $6 \mathrm{~m}$ telescope of Russia and 2.6m telescope of Byurakan carried out with multipupil spectrographs MPFS and VAGR, correspondently. Processing of the data obtained for more than twenty objects are at different stages (see arXiv:1403.0127 for extended version).
\end{abstract}

